\title{
Tobacco specific nitrosamines and potential reduced exposure products for smokers: a preliminary evaluation of Advance ${ }^{\mathrm{TM}}$
}

\author{
A B Breland, M C Acosta, T Eissenberg
}

Tobacco Control 2003;12:317-321

See end of article for authors' affiliations

\section{Correspondence to:}

Thomas Eissenberg, PhD,

Department of Psychology and Institute for Drug and Alcohol Studies, Virginia Commonwealth University, Box 980205, Richmond, VA 23298-0205, USA;

teissenb@vcu.edu

Received

10 January 2003

Accepted 11 June 2003

\begin{abstract}
Objective: To develop a method for evaluating the carcinogen delivery of potential reduced exposure products (PREPs) like Advance ${ }^{T M}$, a PREP marketed to reduce smokers' exposure to one tobacco specific nitrosamine (TSN), NNK, a potent lung carcinogen.

Design, setting, and participants: Latin square ordered, three condition, outpatient, crossover design with 12 smokers of light or ultra-light cigarettes (15 or more cigarettes/day). In each five day condition, participants either smoked own brand, Advance ${ }^{\mathrm{TM}}$, or no cigarettes. Also, on the first and last day of each condition, participants smoked one cigarette in the laboratory.

Main outcome measures: Subject rated measures of tobacco/nicotine withdrawal, expired air carbon monoxide, urine concentrations of cotinine and NNAL (one TSN biomarker), puff volume, duration, number, and interpuff interval.

Results: Relative to own brand, Advance ${ }^{\mathrm{TM}}$ produced similar withdrawal suppression, slightly lower carbon monoxide, equivalent cotinine, and 51\% lower NNAL concentrations. The lowest cotinine and NNAL concentrations were observed in the no cigarette condition. Participants took fewer puffs when smoking Advance ${ }^{\mathrm{TM}}$.

Conclusions: Past experience with PREPs that failed to reduce smoking's harm demonstrates the need for clinical methods in PREP evaluation. This study shows how assessing PREP induced changes in withdrawal and exposure to carbon monoxide, nicotine, and carcinogens may help predict PREP harm reduction potential. Adequate withdrawal suppression, slightly lower concentrations of carbon monoxide, and reduction of one TSN biomarker were observed for Advance ${ }^{\mathrm{TM}}$. In the future, clinical methods like those described here may be valuable for evaluating PREPs before they are marketed publicly.
\end{abstract}

$\mathrm{T}$ he causal relation between tobacco smoking and a variety of cancers (for example, lung, larynx, pancreas, bladder, etc) is well documented ${ }^{1}$ and is attributable to the many carcinogens that smokers inhale, including tobacco specific nitrosamines (TSNs). ${ }^{2}$ The best method for smokers to avoid these tobacco delivered carcinogens is to quit smoking. However, most smokers find quitting difficult because they are dependent upon cigarette delivered nicotine. ${ }^{3}$ As an alternative, the tobacco industry markets potential reduced exposure products (PREPs) that purport to reduce smokers' tobacco delivered carcinogen exposure (for example, RJ Reynolds' Eclipse $^{\circledast}$, Brown and Williamson's Advance $\left.{ }^{\mathrm{TM}}\right) .{ }^{45}$ Careful evaluation of these and other PREPs is essential, ${ }^{56}$ given that previous industry sponsored PREPs (that is, low yield cigarettes) reduced neither smokers' exposure to tobacco delivered toxicants nor tobacco associated mortality. ${ }^{7}$

PREP evaluation will likely be complex, involving the combined efforts of basic, epidemiological, and clinical researchers. Basic research studies can reveal carcinogen concentrations within tobacco and/or tobacco smoke, though they do not model the variability of human smoking behaviour. ${ }^{78}$ Epidemiological studies can reveal the extent to which PREPs increase or decrease the risks of tobacco use, ${ }^{7}$ though data can take years to accumulate. Short term clinical studies are efficient and valuable, and can reveal PREP effects on tobacco use parameters (for example, puff volume or number), withdrawal symptomatology, and exposure to carbon monoxide (CO) and nicotine. ${ }^{45}$ However, some biomarkers for tobacco carcinogen exposure have long half lives, ${ }^{9}$ requiring longer term clinical studies to assess PREP induced changes in carcinogen exposure. $^{4-6}$
Advance $^{\mathrm{TM}}$ is a PREP marketed as a means to reduce exposure to tobacco toxicants, including TSNs. ${ }^{10}$ According to the manufacturer, "a patented tobacco curing process significantly inhibits the formation of tobacco-specific nitrosamines" in the tobacco used to make this PREP. ${ }^{10}$ No epidemiological data are available regarding Advance ${ }^{\mathrm{TM}}$. In a short term clinical study, Advance ${ }^{\mathrm{TM}}$ produced equivalent withdrawal suppression and tachycardia, $11 \%$ lower CO, and a $25 \%$ increase in plasma nicotine, relative to own brand cigarettes. ${ }^{4}$ TSN exposure was not examined, because smokers only used Advance ${ }^{\mathrm{TM}}$ in a single, 2.5 hour session. Longer evaluations will be required to characterise PREP induced changes in TSN exposure. For example, one TSN thought to play a role in lung cancer in smokers is 4-(methylnitrosamino)-1-(3-pyridyl)-1-butanone (NNK). ${ }^{26}$ NNK is metabolised to 4-(methylnitrosamino)-1-(3-pyridyl)l-butanol (NNAL). NNAL can be measured in human urine, is present in smokers, and declines during abstinence, with a distribution half life of 3.4 days. ${ }^{9}$ Thus, clinical evaluation of PREPs like Advance ${ }^{\mathrm{TM}}$ will likely involve measuring urinary NNAL concentration across several days of PREP use.

This preliminary study was designed to determine whether or not clinical methods can be used to measure PREP induced changes in urine concentrations of carcinogen biomarkers.

Abbreviations: FTC, Federal Trade Commission; IPI, interpuff interval; NNAL, 4-(methylnitrosamino)-1-(3-pyridyl)-1-butanol; NNK, 4-(methylnitrosamino)-1-(3-pyridyl)-1-butanone; PREP, potential reduced exposure product; TSN, tobacco specific nitrosamine; QSU, questionnaire of smoking urges, VAS, visual analogue scale 
Because of the preliminary nature of the current study, smokers' exposure to one carcinogen (NNK) was chosen for evaluation for three reasons: (1) NNK is a potent lung carcinogen ${ }^{2}$; (2) several PREPs that are currently marketed in the US purport to reduce smokers' exposure to NNK; and (3) measurement of NNAL, an NNK metabolite, is well validated. ${ }^{9}$ Participants completed three, five day, outpatient conditions, in which they smoked no cigarettes, their own brand, or Advance $^{\mathrm{TM}}$ cigarettes ad libitum. Urine samples were collected on days 1, 3, and 5 of each condition. An important goal of the study was to compare NNAL concentration in smokers' urine across the three conditions; we also measured Advance ${ }^{\mathrm{TM}} \mathrm{s}$ effects on CO, cotinine, tobacco/nicotine withdrawal symptoms, and, in a laboratory evaluation on the first and last day of each condition, puff topography.

\section{DESIGN AND METHODS \\ Participants and setting}

Eight women (one non-white) and four men (one non-white) completed this institutional review board (IRB) approved study. Participants were included if they were 18-50 years old (mean (SD) 24 (8.4) years), provided a breath sample $\geqslant 15$ parts per million (ppm) CO at screening (mean 25.3 (9.7) ppm), and smoked $\geqslant 15$ king sized, non-mentholated, "light" or "ultra-light" cigarettes/day (mean 18.8 (2.7) cigarettes/ day). They were moderately nicotine dependent, as indicated by the Fagerstrom ${ }^{11}$ nicotine tolerance questionnaire (mean 4.9 (0.9)). Exclusion criteria included past or current cardiovascular disorders and current pregnancy, breastfeeding, or smoking cessation or reduction efforts.

\section{Materials}

Depending upon condition, participants smoked own brand (Own), Advance ${ }^{\mathrm{TM}}$ (Adv), or no cigarettes. Participants' Own cigarettes were Marlboro Lights (seven participants), Camel Lights (three participants), Doral Lights (one participant), and Marlboro Ultra Lights (one participant). By the Federal Trade Commission (FTC) method, ${ }^{12}$ on average, Own yielded $0.75 \mathrm{mg}$ nicotine, $11.3 \mathrm{mg} \mathrm{CO}$, and $10.3 \mathrm{mg}$ tar. Similarly, by the FTC method, Adv yielded $0.8 \mathrm{mg}$ nicotine, $9.1 \mathrm{mg} \mathrm{CO}$, and $9.8 \mathrm{mg} \operatorname{tar}^{10}{ }^{10}$ In all cases, $\mathrm{Adv}$ and Own were equal length $(85 \mathrm{~mm})$. However, as described, the tobacco used in Advance $^{\mathrm{TM}}$ cigarettes is cured such that, according to the manufacturer, formation of TSNs is inhibited..$^{10}$ According to the FTC method, smoke from Advance ${ }^{\mathrm{TM}}$ cigarettes contains $80 \%$ less NNK than smoke from normally marketed cigarettes. ${ }^{10}$ All cigarettes used in this study were purchased by laboratory staff and re-packaged into containers with no product labelling.

\section{Compliance measures}

Because the study involved five days of no smoking, compliance with all study conditions was monitored using a combination of daily expired air CO (BreathCO, Vitalograph, Lenaxa, Kansas, USA) and thrice weekly semi-quantitative analysis of cotinine (a nicotine metabolite) in urine (using Nicalert ${ }^{\circledR}$ test strips; Nymox, Maywood, New Jersey, USA).

\section{Main outcome measures}

Daily computerised withdrawal measures consisted of visual analogue scales (VAS) and the questionnaire of smoking urges (QSU). VAS items were presented above a horizontal line with anchors on the left ("not at all") and right ("extremely"). Subjects moved a mouse controlled cursor and clicked to produce a vertical mark on the horizontal line. The score was the distance of the vertical mark from the left anchor, expressed as a percentage of line length. VAS items describe tobacco/ nicotine withdrawal symptoms: "Urges to smoke", "Irritability/frustration/anger", "Anxious", "Difficulty concentrating", "Restlessness", "Hunger", "Impatient", “CRAVING a cigarette/nicotine", "Insomnia/disturbed sleep", "Increased eating", "Drowsiness", "Depression/feeling blue", and "Desire for sweets". ${ }^{5}$ The QSU consists of 32 multiple choice items, and yields two empirically derived factors: factor 1 (intention to smoke); and factor 2 (anticipation of relief from withdrawal). ${ }^{13}$

Each day, while the participant rested quietly, heart rate and skin temperature were recorded every 20 seconds for 30 minutes (Monitor 507E, Criticare Systems, Waukesha, Wisconsin, USA). Thrice weekly urine samples were stored at $-70^{\circ} \mathrm{C}$ for later analysis of cotinine $(\mathrm{GC} / \mathrm{MS} ; \mathrm{LOQ}=1 \mathrm{ng} / \mathrm{ml})$ and NNAL total (that is, NNAL and NNAL-glucuronide). NNAL total was measured using LC-MS/MS ( $\mathrm{LOQ}=50 \mathrm{pg} / \mathrm{ml}$; the assay was performed by MDS Pharma Services of Lincoln, Nebraska, USA, and is a modified version of a recently reported $\operatorname{method}^{14}$ ).

Puff topography was assessed while participants smoked a single cigarette on days 1 and 5. Cigarettes were smoked through a mouthpiece connected to a pressure transducer and pressure changes were amplified and digitised. Software (Plowshare Technologies, Baltimore, Maryland, USA) converted signals to airflow $(\mathrm{ml} / \mathrm{s})$ and integrated the data over time for each puff, producing measures of puff number, volume, duration, and interpuff interval (IPI).

\section{Procedure}

Participants completed three, Latin square ordered, five day conditions (Monday to Friday) in which they smoked Own, Adv, or no cigarettes; in all other respects, conditions were identical. Weekends, when participants smoked Own, were washout periods. On each study day, subjective withdrawal, $\mathrm{CO}$, heart rate, and skin temperature were assessed. In addition, a urine sample was obtained on days 1, 3, and 5 . Semi-quantitative urine cotinine was assessed immediately, and separate $3 \mathrm{ml}$ aliquots of urine were stored for later quantitative analysis of cotinine and NNAL. On days 3 and 5, CO and semi-quantitative urine cotinine data were used to assess compliance with condition smoking restrictions. For example, when participants were in the no smoking condition, compliance was verified with decreases in $\mathrm{CO}$ and semi-quantitative cotinine, relative to day 1 , and was reinforced monetarily ( $\$ 30$ on day 3 and $\$ 70$ on day 5) in each condition. Participants who failed to comply with condition restrictions once were offered another chance to complete the condition (two participants repeated the no smoking condition). Participants who failed to comply more than once were withdrawn (one participant was withdrawn for repeated non-compliance in the Adv condition; this participant's data are excluded from all analyses). On days 1 and 5, puff topography was assessed as participants smoked one cigarette (ad libitum) after daily assessments were completed (Own, Adv, or, in the no smoking condition, participants smoked Own). Participants were paid an additional $\$ 100$ for study completion, and thus could earn a total of $\$ 400$ in this three week study.

\section{Data analysis}

The final 15 minutes of heart rate and skin temperature data were averaged to produce a single value for each day. Topography data were treated as in previous work, ${ }^{5}$ yielding average puff number, volume, duration, and IPI for each cigarette. Data were entered into a within-subject condition (Own, Adv, no smoking) by day analysis of variance, which allowed the simultaneous testing of questions related to main effects of condition (that is, "Independent of study day, did outcome measures differ when participants smoked Own, Adv, and/or no cigarettes?"), main effects of day (that is, "Independent of condition, did outcome measures differ across days?"), and the interaction between these two factors (that is, "Did differences in outcome measures observed during each condition depend upon the particular day on which the data were 
Table 1 Results of statistical analyses for all compliance and main outcome measures

\begin{tabular}{|c|c|c|c|c|c|c|}
\hline & \multicolumn{2}{|c|}{ Condition } & \multicolumn{2}{|l|}{ Day } & \multicolumn{2}{|c|}{ Condition by day } \\
\hline & F Value & $\mathrm{p}$ Value & F Value & $\mathrm{p}$ Value & F Value & $\mathrm{p}$ Value \\
\hline \multicolumn{7}{|l|}{ Compliance measures } \\
\hline Expired air $\mathrm{CO}^{*}$ & 37.1 & $<0.01$ & 9.4 & $<0.01$ & 14.0 & $<0.01$ \\
\hline Semi-quantitative cotinine $†$ & 45.5 & $<0.01$ & 27.6 & $<0.01$ & 27.7 & $<0.01$ \\
\hline \multicolumn{7}{|l|}{ Main outcome measures } \\
\hline \multicolumn{7}{|l|}{ Subjective withdrawal* } \\
\hline Urges to smoke & 2.3 & NS & $<1.0$ & NS & 1.6 & NS \\
\hline Irritability/frustration/anger & 3.2 & NS & 1.3 & NS & 2.7 & $<0.05$ \\
\hline Anxious & 6.1 & $<0.01$ & $<1.0$ & NS & 1.5 & NS \\
\hline Difficulty concentrating & 6.2 & $<0.01$ & 1.8 & NS & $<1.0$ & NS \\
\hline Restlessness & 2.7 & NS & $<1.0$ & NS & 1.7 & NS \\
\hline Hunger & 4.1 & $<0.05$ & $<1.0$ & NS & 1.7 & NS \\
\hline Impatient & 6.9 & $<0.01$ & 1.7 & NS & 1.0 & NS \\
\hline CRAVING a cigarette/nicotine & 3.7 & NS & 1.4 & NS & 1.5 & NS \\
\hline Insomnia/disturbed sleep & $<1.0$ & NS & $<1.0$ & NS & 1.4 & NS \\
\hline Increased eating & 9.5 & $<0.01$ & 4.2 & $<0.01$ & 3.7 & $<0.01$ \\
\hline Drowsiness & 2.0 & NS & 3.2 & $<0.05$ & 1.3 & NS \\
\hline Depression/feeling blue & 5.9 & $<0.01$ & 1.5 & NS & 1.1 & NS \\
\hline Desire for sweets & 4.5 & $<0.05$ & 2.1 & NS & 4.2 & $<0.01$ \\
\hline QSU factor 1 & 6.4 & $<0.05$ & 1.0 & NS & $<1.0$ & NS \\
\hline QSU factor 2 & 9.8 & $<0.01$ & 1.6 & NS & 3.5 & $<0.01$ \\
\hline \multicolumn{7}{|l|}{ Physiological measures* } \\
\hline Heart rate & 15.0 & $<0.001$ & 2.8 & $<0.05$ & 3.6 & $<0.01$ \\
\hline Skin temperature & $<1.0$ & NS & $<1.0$ & NS & $<1.0$ & NS \\
\hline Quantitative cotinine (GC/MS)† & 15.9 & $<0.001$ & 3.4 & NS & 21.1 & $<0.001$ \\
\hline NNAL (LC-MS/MS) $†$ & 11.5 & $<0.001$ & 6.2 & $<0.05$ & 3.7 & $<0.05$ \\
\hline \multicolumn{7}{|l|}{ Puff topography } \\
\hline Puff volume & 2.9 & NS & $<1.0$ & NS & $<1.0$ & NS \\
\hline Puff duration & 1.9 & NS & 1.1 & NS & $<1.0$ & NS \\
\hline Interpuff interval & 2.5 & NS & 1.0 & NS & $<1.0$ & NS \\
\hline Number of puffs & 8.5 & $<0.05$ & $<1.0$ & NS & 2.1 & NS \\
\hline $\begin{array}{l}* d f=8,88 \\
\dagger d f=4,44 \\
\ddagger d f=2,16\end{array}$ & & & & & & \\
\hline
\end{tabular}

collected?"). A complete description of the theory and computation of the analysis of variance (ANOVA) statistical procedure can be found in several authoritative texts ${ }^{15}{ }^{16}$ and is beyond the scope of this work. For CO, subjective withdrawal, heart rate, and skin temperature there were five levels of day (day 1-5); for urine cotinine and NNAL, there were three levels of day (days 1, 3, and 5); and for puff topography there were two levels of day (days 1 and 5). Significance levels were adjusted for violations of the sphericity assumption using Huynh-Feldt corrections. Paired $t$ tests were used to examine mean differences between conditions on each day for measures with significant interactions and/or a condition main effect; because these comparisons were planned in this preliminary evaluation, results with a probability value of $\mathrm{p}<0.05$ are reported as significant. ${ }^{15}$

\section{RESULTS}

Statistical analysis results for main effects of condition, day, and the interaction of condition and day are shown in table 1. A significant effect of condition indicates that outcome measures differed, depending on condition (Own, Adv, or no smoking). A significant effect of day indicates that outcome measures changed significantly over time (from day 1 to day 5 ). A significant condition by day interaction indicates that differences in outcome measures among conditions depended on the day the outcomes were assessed. Main effects of condition and interactions of condition by day are of greatest interest, as these results indicate outcomes that depended upon Own, Adv, and/or no smoking.

\section{Compliance measures}

Table 1 shows a significant condition by day interaction for $\mathrm{CO}$ and semi-quantitative urine cotinine. The mean CO concentrations obtained on each day of each condition ( \pm I SEM) are displayed in fig $\mathrm{l}$ (top panel). Mean CO concentrations on days $2-5$ of the no smoking condition were significantly lower than Own and Adv. Interestingly, relative to Own, mean (SD) CO concentrations were significantly lower in the $\mathrm{Adv}$ condition on day 2 (Own 22.0 (10.0) ppm; Adv 15.2 (10.8) ppm), day 4 (Own 20.6 (11.2) ppm; ADV 16.6 (8.3) ppm), and day 5 (Own 21.3 (9.2) ppm; Adv 15.0 (10.8) ppm). For semi-quantitative urine cotinine (values range from 0-6), mean (SD) concentrations on day $3(5.1(1.1))$ and day 5 of the no smoking condition (3.0 (1.4)) were significantly lower as compared to Own and Adv (Own day 3, 5.9 (0.3); Own day 5, 6.0 (0.0); Adv day 3, 5.9 (0.3); Adv day 5, 5.9 (0.3) data not shown). No differences in semi-quantitative urine cotinine were observed between Own and Adv.

\section{Main outcome measures}

Regarding subjective withdrawal, scores increased in the no smoking condition relative to Own and Adv across study days. For example, for the "Desire for sweets" VAS (the measure with the largest interaction F value), scores in all three conditions were similar on day 1 , but on day 2 scores in the no smoking condition (mean (SD) 31.4 (33.0)) were significantly greater than for Own (14.3 (29.0)) and Adv (16.3 (28.8)). For this measure on day 5 , mean scores in the no smoking condition (37.2 (39.5)) were significantly higher relative to Own 

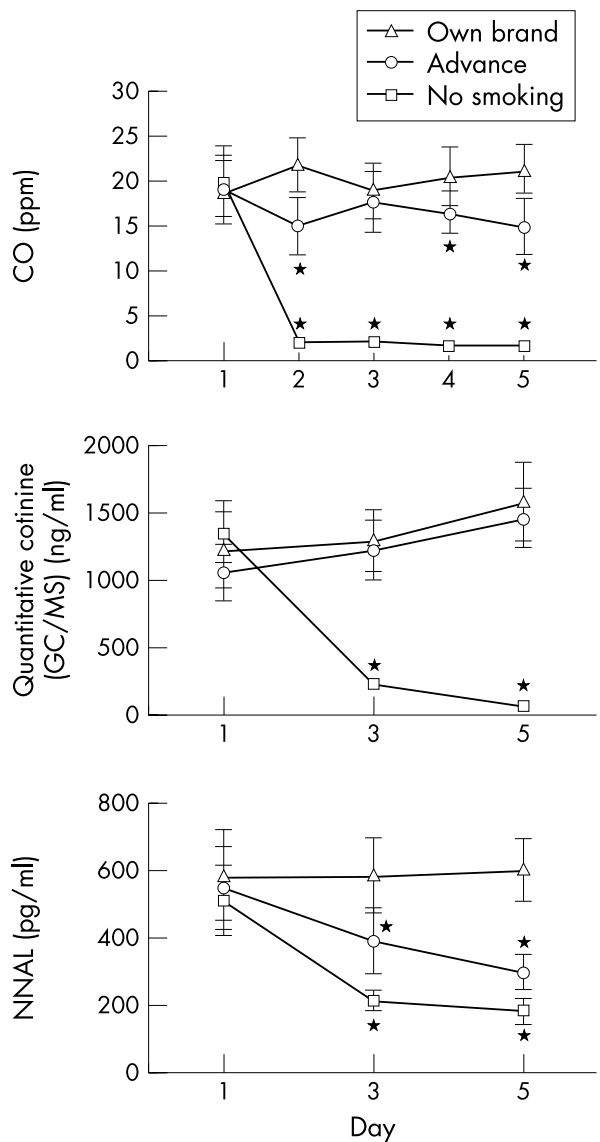

Figure 1 Averaged data (plus 1 SEM) from 12 subjects for expired air carbon monoxide (top), urine cotinine concentration (middle), or urine NNAL total concentration (bottom) across the five days of each single blind condition. * Indicates a significant difference from own brand at that time point (all $p<0.05$ ).

(15.1 (29.0)) and were elevated relative to Adv (18.2 (28.2); $\mathrm{p}<0.08$ ). A similar pattern of results (higher ratings on study days 2-5 for no smoking relative to Own and Adv) was observed on the other subjective withdrawal measures with significant main effects or interactions (that is, "Irritability/ frustration/anger", "Anxious", "Difficulty concentrating", "Hunger", "Impatient", "Increased eating", "Depression/ feeling blue", and both factors of the QSU.)

Table 1 also shows a significant condition by day interaction for heart rate and no main effects for interaction for skin temperature. Mean heart rate in the no smoking condition was significantly lower relative to Own on day 3 (no smoking mean (SD) 69.0 (9.1) bpm; Own 82.1 (6.0) bpm), day 4 (no smoking 72.8 (9.5) bpm; Own 80.3 (7.5) bpm), and day 5 (no smoking 75.0 (9.5) bpm; Own 82.9 (8.5) bpm), but was significantly lower than Adv on days 3 (78.5 (7.8) bpm) and 4 (80.0 (9.2) bpm) only.

Quantitative urine cotinine and NNAL concentrations depended on both condition and day. Figure 1 (middle panel) shows that, as expected, mean quantitative cotinine concentrations were similar on day 1 (recall that participants smoked Own during the minimum 72 hour washout period that separated each condition, thus biomarkers associated with smoking should be equally high after each washout period), but were significantly lower in the no smoking condition relative to Own and Adv on day 3 (no smoking mean (SD) 214.0 (180.0) ng/ml; Own 1283.6 (815.0) ng/ml; Adv 1221.1 (75l.4) ng/ml), and day 5 (no smoking 54.1 (58.7) ng/ml; Own 1571.8 (1013.6) ng/ml; Adv 1448.8 (747.2) ng/ml). No differences in quantitative urine cotinine were observed between Own and Adv on any day.
Figure l (bottom panel) shows a somewhat different pattern for NNAL. There were no between condition differences on day 1, but on days 3 and 5, NNAL concentrations were significantly lower in the no smoking and Adv conditions, relative to Own (for day 3: no smoking mean (SD) 213.2 (109.1) pg/ml; Adv 394.6 (338.0) pg/ml; Own 588.8 (398.5) pg/ml; for day 5, no smoking 182.2 (131.5) pg/ml; Adv 298.2 (183.6) pg/ml; Own 603.9 (319.0) pg/ml).

Participants smoked a single cigarette in the laboratory on days 1 and 5 of each condition. For puff number, a significant effect of condition was observed. Participants took fewer puffs, on average, from Adv (mean (SD) for day 1, 9.6 (2.8) puffs; day 5, 9.2 (2.9) puffs) than from Own (day 1, 11.7 (4.2) puffs; day 5, 10.8 (3.0) puffs). No significant effects were observed for puff volume, puff duration, or interpuff interval, indicating that decreased puff number was the only behavioural change observed when participants smoked Adv, relative to when they smoked Own.

\section{DISCUSSION}

Cessation is the only proven method for tobacco users to decrease their exposure to tobacco related carcinogens, and thus reduce their likelihood of tobacco related disease and death. Advance ${ }^{\mathrm{TM}}$ is intended to reduce smokers' exposure to TSNs, including NNK, one potent lung carcinogen. However, the link between reduced TSN exposure and decreased death and disease is uncertain, and there are no accepted methods for demonstrating that Advance ${ }^{\mathrm{TM}}$ (or any cigarette-like PREP) actually reduces smokers' carcinogen exposure. This study was designed to examine clinical methods of measuring PREP induced changes in carcinogen exposure, by comparing TSN metabolite concentrations (that is, urine NNAL) when smokers used their own brand, Advance ${ }^{\mathrm{TM}}$, or no cigarettes for five days.

As might be expected, smoking own brand cigarettes maintained urine NNAL concentrations (that is, a $2.1 \%$ increase in mean NNAL was observed) across study days. Relative to own brand, by the fifth day, Advance ${ }^{\mathrm{TM}}$ use was associated with $51 \%$ lower mean NNAL concentrations, while not smoking was associated with $70 \%$ lower concentrations (fig 1, bottom panel). These data demonstrate that PREP induced changes in carcinogen exposure can be measured in a five day clinical study, and suggest that Advance ${ }^{\mathrm{TM}}$ reduces NNK exposure significantly (with longer study periods, even greater reductions in toxicant exposure may be observed). To the extent that TSNs are associated with tobacco related mortality, incorporating low nitrosamine tobacco in other products may be an important public health goal. However, the many other tobacco delivered toxicants that smokers inhale may limit the reduction in death and disease that may be attributable to TSN exposure reduction.

Some other results observed in this study ( small but reliable CO reduction, equivalent withdrawal suppression relative to own brand) are consistent with those reported in a previous short term clinical evaluation of Advance ${ }^{\mathrm{TM}} .{ }^{4}$ Also in that short term study, four, eight puff smoking bouts using Advance ${ }^{\mathrm{TM}}$ produced significantly increased plasma nicotine concentrations, relative to own brand cigarettes. In the current study, Advance $^{\mathrm{TM}}$ and own brand appeared to deliver similar amounts of nicotine, as assessed by urine cotinine concentrations. Importantly, in this study, participants smoked Advance $^{\mathrm{TM}}$ ad libitum and, on average, took 1.8 fewer puffs when using Advance ${ }^{\mathrm{TM}}$ relative to own brand, but did not alter puff volume, duration, or inter-puff interval. This difference in smoking behaviour may explain the apparent discrepancy regarding nicotine intake in these two studies. That is, in the current study, smokers may have received less nicotine from Advance $^{\mathrm{TM}}$ because puff number was free to vary, while puff number was held constant in the earlier work. Thus, puff 
topography is an important outcome measure in PREP evaluation, as PREP induced changes in puff number, volume, duration, and/or inter-puff interval may influence tobacco toxicant exposure. ${ }^{7}$

One potential study limitation is that, when participants were instructed to smoke own brand, Advance ${ }^{\mathrm{TM}}$, or no cigarettes, they may not have complied with these restrictions. Compliance was enhanced with immediate monetary reinforcement based on objective measures of cigarette use (for example, expired air CO, semi-quantitative urine cotinine). CO and quantitative urine cotinine levels (fig l, top and middle panels) are consistent with compliance in the no smoking condition. More sensitive measures may be required to verify complete compliance with all study restrictions. Another potential limitation involves the five day duration of each study condition. Longer periods may allow a more complete characterisation of PREP associated carcinogen exposure and/or changes in smoking topography. For example, according to the manufacturer, NNK concentrations in smoke from Advance $^{\mathrm{TM}}$ are $80 \%$ lower than normally marketed brands. However, in this study, we observed a $51 \%$ reduction in urine NNAL concentrations associated with Advance ${ }^{\mathrm{TM}}$ use. A longer study period may help determine if an $80 \%$ reduction in NNK in cigarette smoke leads to an $80 \%$ reduction in NNAL in smokers' urine. If, over longer study periods, participants increase or decrease the number of puffs that they take when using Advance ${ }^{\mathrm{TM}}$, this behavioural change may also influence NNAL concentrations. Of course, participant compliance with some smoking restrictions may limit condition duration. Finally, one other limitation concerns the possibility of type I and type II error in this preliminary study. Now that a clinical method for assessing PREP induced changes in carcinogen exposure is available, future studies replicating this method with more subjects and/or different PREPs would be valuable.

In summary, previous short term (2.5 hour) clinical PREP evaluations have focused on important measures such as PREP associated CO delivery, nicotine exposure, withdrawal suppression, and physiological response. This longer term study elaborates on the short term clinical PREP evaluation model, by allowing assessment of PREP induced carcinogen exposure. With regard to Advance ${ }^{\mathrm{TM}}$, the current study indicates that PREPs may be able to reduce smokers' tobacco toxicant exposure, suggesting harm reduction potential. Overall, clinical methods have a valuable role to play in PREP evaluation, though a transdisciplinary approach including basic, clinical, and epidemiological research will likely be necessary to demonstrate actual PREP associated harm reduction.

\section{ACKNOWLEDGEMENTS}

This work was supported by USPHS grants R01 DA 011082 and F31 DA015570 as well as the Robert Wood Johnson Foundation's Tobacco Etiology Research Network and VCU's Massey Cancer Center. Portions of this work were presented at the Ninth Annual Meeting of the Society for Research on Nicotine and Tobacco, 19-22 February 2003.

\section{Authors' affiliations}

A B Breland, Department of Psychology, Virginia Commonwealth University, Richmond, Virginia, USA

M C Costa, Departments of Psychiatry and Psychology, Virginia Commonwealth University

\section{What this paper adds}

The tobacco industry has been marketing potential reduced exposure products (PREPs) that purport to reduce smokers' tobacco delivered carcinogen exposure (for example, Brown and Williamson's Advance $\left.{ }^{\mathrm{TM}}\right)$. Few studies describe the effects of PREPs in smokers. Careful evaluation of these and other PREPs is essential, given that previous industry sponsored PREPs (that is, low yield cigarettes) reduced neither smokers' exposure to tobacco delivered toxicants nor tobacco associated mortality.

This study shows how assessing PREP induced changes in withdrawal and exposure to carbon monoxide, nicotine, and carcinogens may help predict PREP harm reduction potential. Adequate withdrawal suppression, slightly lower concentrations of carbon monoxide, and reduction of one tobacco specific nitrosamine biomarker were observed for Advance $^{T M}$. In the future, clinical methods like those described here may be valuable for evaluating PREPs before they are marketed publicly.

T Eissenberg, Department of Psychology and Institute for Drug and Alcohol Studies, Virginia Commonwealth University

\section{REFERENCES}

1 US Department of Health and Human Services. Reducing the health consequences of smoking: 25 years of progress. A report of the Surgeon General, 1989. Rockville, Maryland: Public Health Service, Centers for Disease Control, Office on Smoking and Health, 1989. (DHHS Publication No (CDC) 89-8411.)

2 Hecht S. Tobacco smoke carcinogens and lung cancer. J Ntl Cancer Inst 1999: 91:1194-210.

3 US Department of Health and Human Services. The health consequences of smoking: nicotine addiction. A report of the Surgeon General, 1988. Rockville, Maryland: Public Health Service, Centers for Disease Control, Office on Smoking and Health, 1988. (DHHS Publication No (CDC) 88-8406.)

4 Breland $A B$, Evans SE, Buchhalter AR, et al. Acute effects of Advance ${ }^{T M}$. a potential reduced exposure product for smokers. Tobacco Control $2002 ; 11: 376-8$

5 Breland AB, Buchhalter AR, Evans SE, et al. Evaluating acute effects of potential reduced exposure products for smokers: clinical laboratory methodology. Nicotine and Tobacco Research 2002;4:S131-40.

6 Hecht SS. Human urinary carcinogen metabolites: biomarkers for investigating tobacco and cancer. Carcinogenesis 2002;23:907-22.

7 Stratton K, Shetty P, Wallace R, et al, eds. Clearing the smoke: the science base for tobacco harm reduction. Washington DC: National Academy Press, 2001.

8 Diordjevic MV, Stellman SD, Zang E. Doses of nicotine and lung carcinogens delivered to cigarette smokers. J Ntl Cancer Inst 2000;92:106-19.

9 Carmella SG, Akerkar S, Richie JP Jr, et al. Intraindividual and interindividual differences in metabolites of the tobacco-specific lung carcinogen 4-(methylnitrosamino)-1-(3-pyridyl)-1-butanone (NNK) in smokers' urine. Cancer Epidemiol Biomarkers Prev 1995;4:635-42.

10 Advance $^{\mathrm{Tm}}$ Lights, Kings, pack "onsert" 15-1210. URL: http://www.brownandwilliamson.com/Index_sub2.cfm?ID=6

11 Fagerstrom KO. Measuring degree of physical dependence to tobacco smoking with reference to individualization of treatment. Addictive Behaviors 1978;3:235-41.

12 Federal Trade Commission. "Tar," nicotine, and carbon monoxide of the smoke of 1294 varieties of domestic cigarettes for the year 1998. Washington, DC: FTC, 2000.

13 Tiffany ST, Drobes DJ. The development and initial validation of a questionnaire on smoking urges. Br J Addiction 1991;86:1467-76.

14 Newland K, Grafelman D, Carnes E, et al. LC-MS/MS bioanalysis of total 4-methylnitrosamino-1-(3-pyridyl)-1-butanol (NNAL) in human urine [abstract]. In: Proceedings of the 51 st annual meeting of the American Society of Mass Spectrometry, 8-12 June 2003, Montreal, Quebec.

15 Keppel G. Design and analysis, a researcher's handbook. Englewood Cliffs, New Jersey: Prentice Hall, 1991.

16 Hays WL. Statistics. Fort Worth, Texas: Harcourt Brace College Publishers, 1994. 